\title{
The Immediate Fate of Staphylococci after Phagocytosis
}

\author{
By M. G. SHAYEGANI* AND F. A. KAPRAL \\ The Department of Microbiology, School of Medicine \\ University of Pennsylvania, Philadelphia, U.S.A.
}

(Received 20 February 1962)

\begin{abstract}
SUMMARY
Four methods were employed to study the immediate fate of staphylococci within leucocytes after phagocytosis. These were: $(a)$ The method of Cohn \& Morse where leucocytes and staphylococci were mixed in tubes and kept agitated. Periodically samples were removed and lightly centrifuged to sediment the leucocytes. Plate counts were made on the lysed leucocytes to measure the intracellular organisms, the supernatant to measure the extracellular population, and a non-centrifuged sample to determine the total number of viable cocci present. (b) Concentrated suspensions of leucocytes and staphylococci were packed together by brief centrifugation to permit rapid phagocytosis without antibodies then diluted in cold Hanks solution to stop further phagocytosis. The suspension was then rapidly passed through a Servall centrifuge with the continuous flow attachment to remove excess extracellular organisms. The infected leucocytes were placed in suspension and samples removed and treated as in the previous procedure. (c) Leucocytes and staphylococci were placed in plastic chambers containing a number of small coverslips. The cells were allowed to sediment and adhere to the coverslips, then washed to remove most of the extracellular organisms. Coverslips were removed at intervals and attached leucocytes lysed to liberate intracellular cocci which were enumerated by plate counts. Companion coverslips were washed, fixed, and stained in order to count the number of leucocytes present. The extracellular staphylococcal population was estimated by making plate counts on the tissue culture medium in the chambers. $(d)$ Suspensions of infected leucocytes were diluted and placed in a series of Petri dishes. The cells were permitted to settle and attach to the glass. Periodically the plates were washed and melted trypticase soy agar was added. After incubation the plate counts afforded an estimation of the viable cocci remaining within the leucocytes at each sampling.

The results obtained with these procedures were in fairly good agreement with each other. It was found that Staphylococcus aureus (18-Z and Smith strains) survived in significant numbers within monocytes and polymorphonuclear leucocytes of normal rabbits for several hours. Little destruction of this organism during the first hour after phagocytosis could be demonstrated.
\end{abstract}

* Present address: Department of Clinical Pathology, Philadelphia General Hospital, Philadelphia, Pennsylvania, U.S.A. 


\section{INTRODUCTION}

In previous publications (Kapral \& Shayegani, 1959; Shayegani \& Kapral, 1962) the authors reported that Staphylococcus aureus usually survived for several hours within polymorphonuclear leucocytes and monocytes of normal rabbits, but were eventually slowly destroyed within the monocytes. S. albus, however, was rapidly destroyed by these cells under the same conditions. While these studies were in progress, Cohn \& Morse (1959) reported that $S$. aureus (Smith strain) and $S$. albus were equally rapidly destroyed within rabbit polymorphonuclear leucocytes (over $99 \%$ within $2 \mathrm{hr}$.). Specific rabbit immune serum was used to aid phagocytosis of the organism. Using a similar method, Mackaness (1960) claimed more than $90 \%$ destruction of the Smith strain within rabbit monocytes in $1 \mathrm{hr}$. Rogers \& Melly (1960), however, indicated that $S$. aureus (Smith strain) survived in significant numbers within human polymorphs when phagocytosed in the presence of normal human serum whereas $S$. albus was destroyed. Cohn \& Morse, Mackaness, and Rogers \& Melly, all used the same experimental technique (originally described by Maaløe) and the same organisms, yet their results failed to agree as to the extent by which S. aureus was killed. Melly, Thomison \& Rogers (1960) by direct observation with phase microscopy, demonstrated that $63 \%$ of $S$. aureus (Smith strain) survived 20 min. within human polymorphs; in contrast, $S$. albus was destroyed in this interval. In the latter study all experiments were performed with blood from a single donor.

Since the conflicting results of different investigators could possibly be due to differences in experimental technique or to the use of different strains of Staphylococcus aureus and since our previous studies did not supply any detailed information concerning the fate of staphylococci immediately after phagocytosis, an attempt was made in the present study to apply and compare different methods of studying the fate of staphylococci during this period.

The following procedures were thus designed to study the early phase of intracellular parasitism.

\section{METHODS}

Staphylococcus aureus strains. S. aureus strain $18-\mathrm{Z}$; the characteristics of this strain were described previously (Kapral \& Shayegani, 1959). From this strain two mutants were derived: (i) 18-Z, ad- - an adenine-requiring mutant, which grew slowly without added adenine, and had a lag phase of about $7-10 \mathrm{hr}$.; (ii) $18-\mathrm{Z}, \mathrm{SM}_{\mathrm{d}}-\mathrm{a}$ streptomycin-dependent mutant with a moderately high frequency of backmutation; about 1 in 60,000 can grow in the absence of streptomycin. Populations of this strain show no significant increases in streptomycin-free medium for at least $10 \mathrm{hr}$. These mutants appeared identical with the parent strain in all other in vitro characteristics.

Staphylococcus aureus strain Smith: was obtained through the courtesy of Drs Z. A. Cohn and S. I. Morse of the Rockefeller Institute, New York.

Leucocytes. Monocytes and polymorphonuclear leucocytes were obtained from rabbits as described previously (Kapral \& Shayegani, 1959). In some experiments polymorphs were obtained by injecting $30 \mathrm{ml}$. of $0.1 \%$ glycogen in saline intraperitoneally into rabbits a day before, and again $3 \mathrm{hr}$. before collection of the cells. 


\section{Tissue-culture procedures}

Tissue-culture chamber procedure. Described in detail in Kapral \& Shayegani, (1959).

Method of Cohn \& Morse. As described by Cohn \& Morse (1959) with the following modifications: (a) the tubes were incubated in a roller drum ( $26 \mathrm{cyc} . / \mathrm{min}$.$) at 37^{\circ}$; $(b)$ the leucocytes were disrupted with $5 \%$ sterile saponin solution; $(c)$ viable leucocyte counts were also done on each sample using $1 \%$ trypan blue.

Suspended culture methods. Cells were maintained in siliconized $50 \mathrm{ml}$. Erlenmeyer flasks which had been altered so as to have a side port. The cells were kept suspended by means of Teflon-covered stirring bars rotated (30 r.p.m.) by a series of magnets connected with a belt driven by a variable speed motor. The flasks were continually gassed with $5 \% \mathrm{CO}_{2}$ in air. Sodium bicarbonate was added to the medium to give a $\mathrm{pH}$ of $\mathbf{7 \cdot 2}$ under this atmosphere. Samples were removed at desired intervals through the side port.

Monocytes or polymorphonuclear leucocytes were induced in rabbits in the same manner already described, the peritoneal cavity was washed with $100 \mathrm{ml}$. Hanks solution containing heparin and $2 \%$ normal rabbit serum. This suspension was passed through four layers of sterile gauze and then centrifuged at $250 \mathrm{~g}$ for $10 \mathrm{~min}$. The sediment was resuspended in $2 \mathrm{ml}$. Hanks solution containing $10 \%$ normal rabbit serum. Leucocyte counts were made on a diluted sample of this suspension. The leucocyte suspension was mixed with a suspension of washed staphylococci (ratio of 25 cocci per leucocyte) in a conical centrifuge tube, and was packed by centrifugation at $100 \mathrm{~g}$ for $3 \mathrm{~min}$., to permit rapid phagocytosis without requiring antibodies. The supernate was decanted, the sediment was resuspended in 1 l. of cold Hanks solution and rapidly passed through a continuous flow centrifuge (Servall) with a two-channel distributor at 7700g, to eliminate most of the extracellular organisms. One litre of infected cell suspension would pass through the centrifuge in 3-4 min. The sedimented leucocytes from the two centrifuge tubes were pooled in $1 \mathrm{ml}$. Hanks solution with $10 \%$ normal rabbit serum and transferred to the culture flask containing 10-12 ml. medium (10\% normal rabbit serum in Hanks solution).

After mixing, the zero-time sample was removed from the flask with a $1 \mathrm{ml}$. pipette. The entire process from the time the staphylococci were added to the leucocytes to removal of zero time samples from the flask required 18-20 min.

Samples at various intervals were treated as follows: one half $\mathrm{ml}$. of sample was transferred into $4.5 \mathrm{ml}$. cold saline in a Wassermann tube, centrifuged at $50 \mathrm{~g}$ for $4 \mathrm{~min}$. Then $0.5 \mathrm{ml}$. of the supernatant was diluted and plated on trypticase soy agar to measure the extracellular staphylococcal population. The remaining supernatant was discarded. The sedimented cells were lysed with $1 \mathrm{ml}$. of $5 \%$ sterile saponin solution in order to release the intracellular organisms. This lysed cell preparation was also diluted and plate counts made. Two-tenth ml. of the sample was also transferred into $0.8 \mathrm{ml}$ of cold $5 \%$ saponin in another Wassermann tube, mixed, diluted, and plated out to enumerate the total number of organisms in the system. One-tenth $\mathrm{ml}$. of the sample was mixed with $\mathbf{0 . 2} \mathrm{ml}$. of $1 \%$ trypan blue to count viable leucocytes.

Plate methods. The leucocytes and organisms were packed in a conical tube as 
previously described and kept in a water bath at $37^{\circ}$ for $15 \mathrm{~min}$. The supernatant was decanted and the sediment washed three times with $100 \mathrm{ml}$. cold Hanks solution to remove most of the extracellular organisms. In some experiments the infected cell suspensions prepared for the suspended cultures were used.

The infected cell suspension was diluted in Hanks solution. Five ml. samples were distributed to a series of Petri dishes. The cells settled on the bottom of the dishes at $37^{\circ}$ for $15 \mathrm{~min}$. and were then washed twice with $5 \mathrm{ml}$. quantities of Hanks solution, melted trypticase soy agar was added to some dishes immediately (zero time). The remaining Petri dishes received $5 \mathrm{ml} .10 \%$ normal rabbit serum in Hanks solution and were kept at $37^{\circ}$.

At different intervals, dishes were washed twice with Hanks solution and melted trypticase soy agar was added. The zero time was normally some $90 \mathrm{~min}$. after initial phagocytosis of organisms by leucocytes. By this method, if intracellular killing of organisms should occur during the period of observation, plate counts at intervals should decrease, provided that the population of leucocytes per Petri dish remained unchanged.

Continued phagocytosis of extracellular organisms would also give the impression that killing was occurring, but since such small numbers were used, the possibility of this phenomenon is remote.

\section{RESULTS}

The first logical approach was to check whether the intracellular behaviours of the Smith strain and 18-Z strains were different. The method described by Cohn \& Morse was used to follow this.

Strain 18-Z and rabbit monocytes. Figure 1 illustrates the results of such an experiment in which $10 \%$ normal and specific immune sera were used. It appeared that the decline of the total bacterial count of the 18-Z strain was comparable to that of the Smith strain as reported by Mackaness (1960).

Strain 18-Z and rabbit polymorphs. A series of experiments was performed to follow the intracellular survival of $18-\mathrm{Z}$ in rabbit polymorphs using normal rabbit serum. At the same time it was decided to see if repeated injections of glycogen intraperitoneally into rabbits might perhaps change the activity of the leucocytes from the corresponding exudates. Therefore, $150 \mathrm{ml}$. of $0 \cdot 1 \%$ glycogen in pyrogenfree saline was injected intraperitoneally, daily into a group of rabbits. Four hr. after the glycogen injection' $100-150 \mathrm{ml}$. of Hanks solution with heparin $(1 / 20,000)$ was injected into the peritoneal cavity and the exudate collected through a trocar. Figure 2 illustrates data obtained with polymorphs from a representative rabbit. It can be seen that the intracellular survival of $18-\mathrm{Z}$ in rabbit polymorphs is comparable to that observed in rabbit monocytes, but is quantitatively different from that reported for the Smith strain by Cohn \& Morse (1959). Furthermore, although the number of polymorphs obtainable in the exudate increased with repeated glycogen stimulation, the intracellular behaviour of the organisms appeared to remain essentially the same in cells from the same rabbit.

Smith strain in rabbit polymorphs. The survival of the Smith strain within polymorphs from different rabbits was studied with specific immune serum. Figure 3 illustrates the result of such an experiment. The survival of the Smith strain within 
polymorphs was similar to that observed with the 18-Z strain within polymorphs and monocytes. It was not possible to demonstrate the rapid and extensive destruction of the organisms as reported by Cohn \& Morse (1959).

An attempt was also made to follow the fate of the 18-Z and Smith strains within rabbit monocytes shortly after phagocytosis by using the plastic chamber method previously described. Here, only $15 \mathrm{~min}$. were allowed for the settling of the monocytes on to the coverslip; the zero time samples were actually taken some $20 \mathrm{~min}$. after the initial contact of monocytes and organisms. These experiments were performed with normal rabbit serum in the absence of antibiotics. Figure $4 \mathrm{~A}$ shows the results of such an experiment in which there was no apparent destruction of the intracellular 18-Z strain and Smith strain up to $3 \mathrm{hr}$. It also confirmed that the $18-\mathrm{Z}$ strain was phagocytosed in normal rabbit serum while the Smith strain could resist phagocytosis under the same conditions.

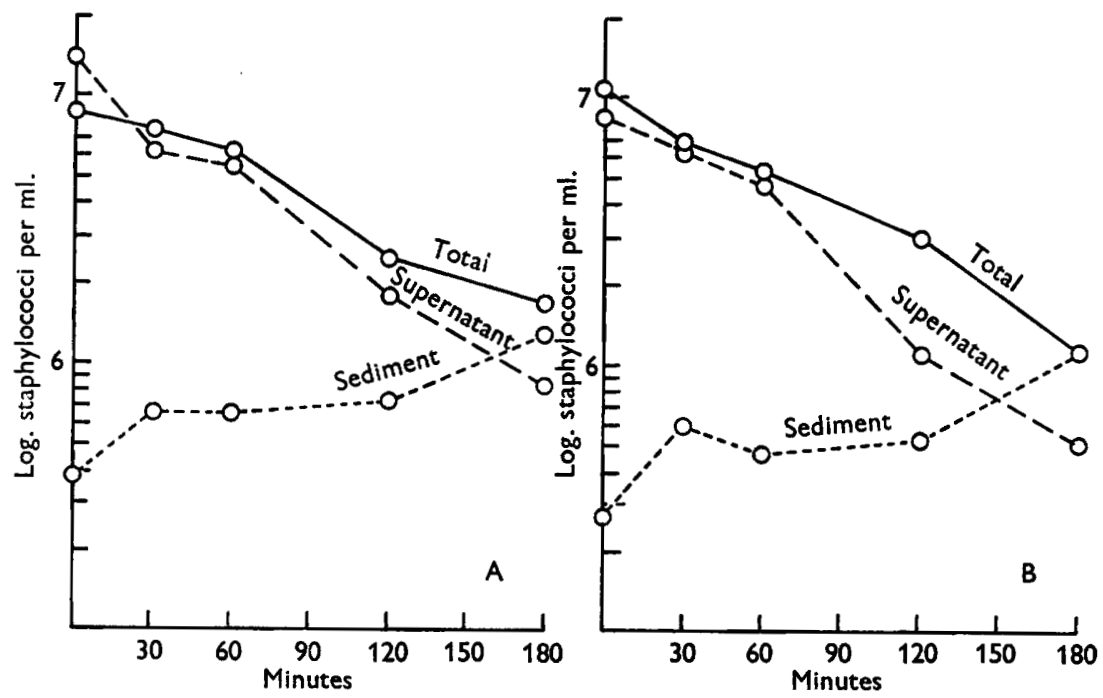

Fig. 1. The survival of Staphylococcus aureus $18-\mathrm{Z}$ within rabbit monocytes. A. In the presence of normal rabbit serum. B. In the presence of specific immune rabbit serum.

Packing the leucocytes and organisms by centrifugation (described under the plate method) was found to be effective in aiding the initial phagocytosis of the Smith strain. This avoided the use of antiserum whose presence might interfere with the results, hence the packing method in the presence of normal rabbit serum was routinely used. Figure $4 \mathrm{~B}$ illustrates the result of such an experiment which was performed in the presence of $20 \mu \mathrm{g}$. streptomycin $/ \mathrm{ml}$. medium, and using an $18 \mathrm{hr}$. old culture of the Smith strain. Here there is some indication of slight intracellular destruction of the organism, but this does not appear to be dependent upon specific antibodies. Perhaps this slight destruction is in part due to the presence of streptomycin, since the Smith strain is more sensitive to the antibiotic than is the 18-Z strain.

In order to follow the intracellular survival of staphylococci for a somewhat longer period, without resorting to antibiotics as a means of preventing extracellular multiplication, experiments were also performed using the adenine-requiring and strepto- 
mycin-dependent mutants of strain 18-Z. Monocytes were infected with these mutants and placed in plastic chambers in the absence of any antibiotics. Figure 5 presents data from such studies over a $10 \mathrm{hr}$. period. There is no evidence of any significant destruction of these mutants within monocytes during this interval.

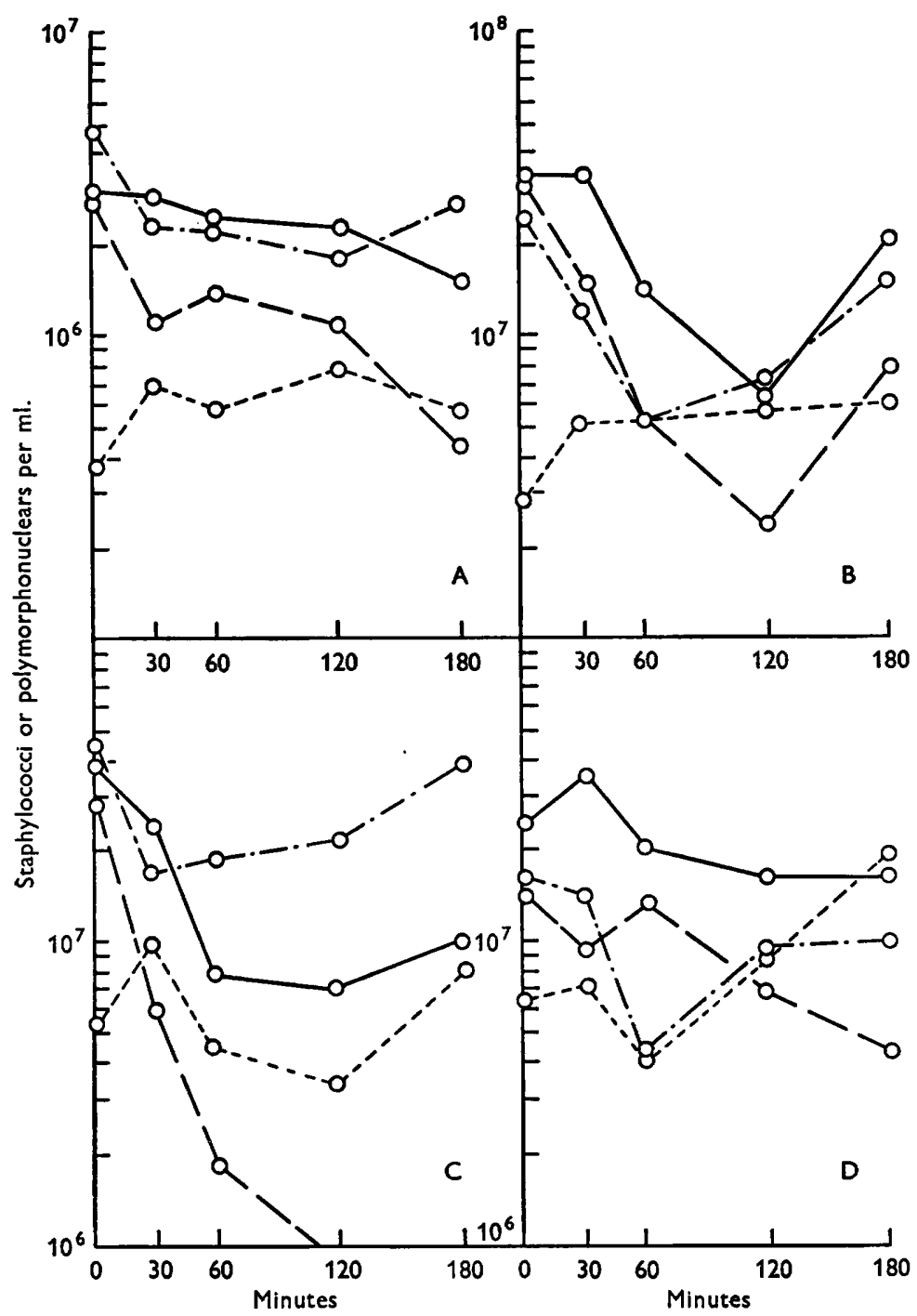

Fig. 2. Intracellular survival of Staphylococcus aureus 18-Z in rabbit polymorphs obtained $4 \mathrm{hr}$. after the first (A), third (B), fifth (C), and eighth (D) daily intraperitoneal glycogen injection. - Total; -.- sediment; - - supernatant; - - - - polymorphs.

The plate method offered a different approach to this problem. Rabbit polymorphs and monocytes were infected with the 18-Z and Smith strains as described under Methods. The results are illustrated in Fig. 6 in which there appeared to be little, if any, intracellular destruction of the $18-\mathrm{Z}$ or Smith strain within rabbit polymorphs or monocytes during the period of observation. 


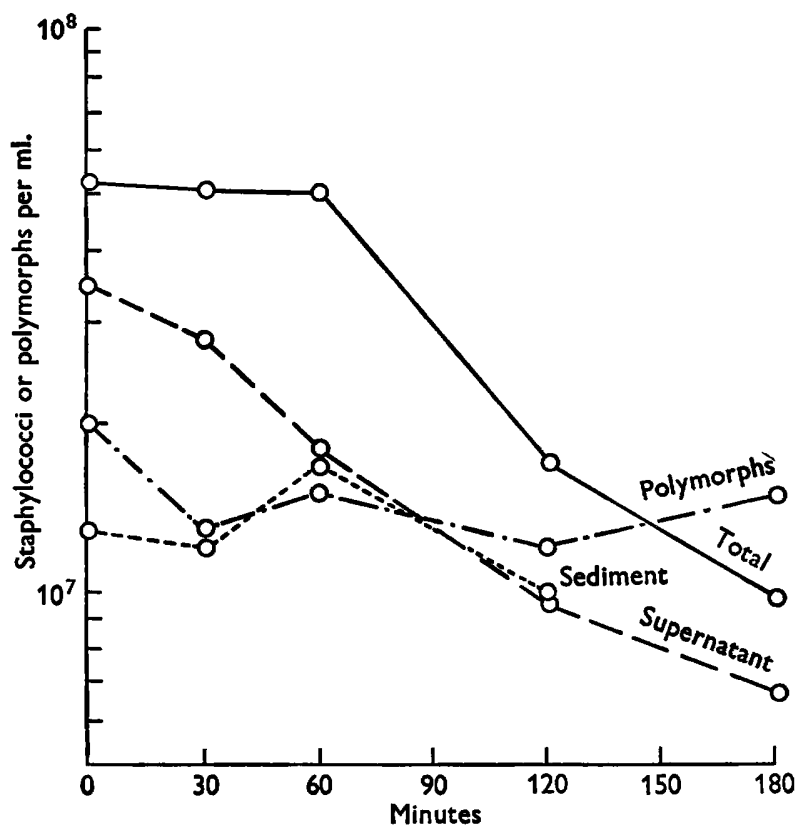

Fig. 3. The intracellular survival of Staphylococcus aureus Smith within rabbit polymorphs in the presence of specific antiserum.

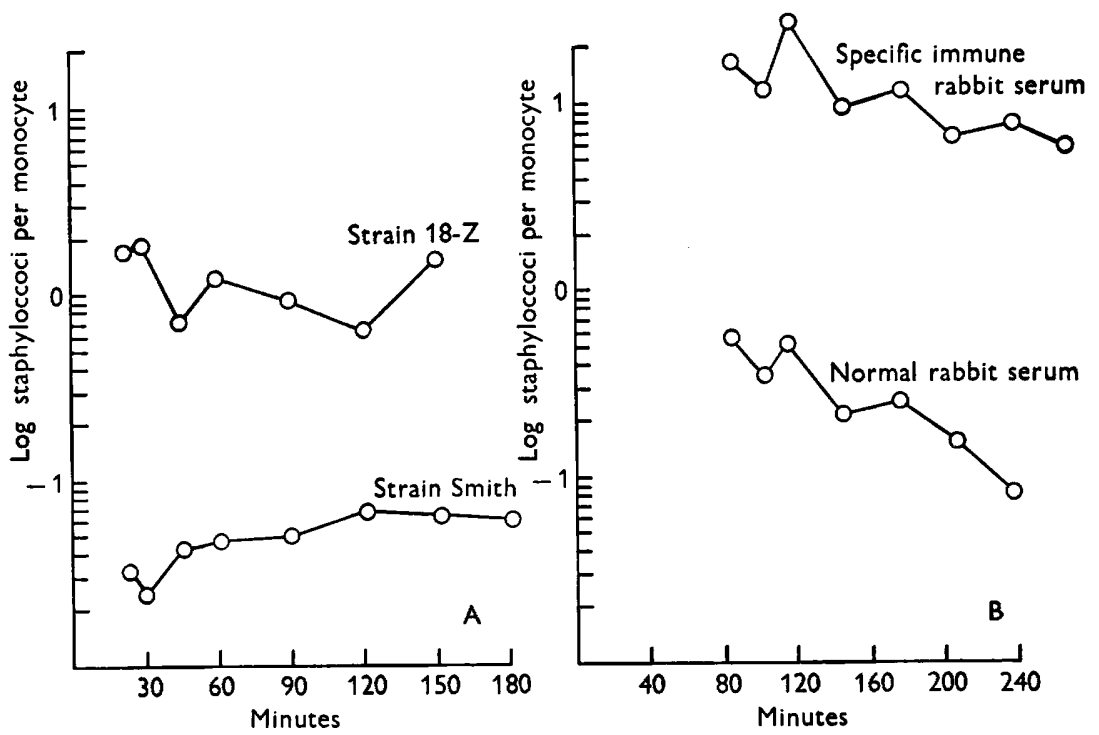

Fig. 4. A. Intracellular survival of Staphylococcus aureus Smith and 18-Z within rabbit monocytes (no antibiotic in medium). B. Intracellular survival of $S$, aureus Smith within rabbit monocytes in the presence of normal and immune rabbit serum $(20 \mu \mathrm{g}$. streptomycin/ml. medium). 
The suspended tissue culture method was also employed to study the immediate fate of intracellular staphylococci. In some experiments, the period of observation was extended up to $10 \mathrm{hr}$. With this method, the intracellular, extracellular, and total population of organisms as well as the viable leucocytes could be followed. With the Cohn \& Morse method the rapid decline of the total population and the extracellular organisms was interpreted as the combined effect of continuous phagocytosis and intracellular destruction. In order to eliminate continuous phagocytosis of extracellular organisms which might complicate determining the fate of intracellular organisms, it would be desirable to remove as many extracellular staphylococci as possible subsequent to initial infection of the cells. The continuous flow centrifugation procedure as described under Methods was used and the period from initial phagocytosis to removal of zero time samples was reduced to $18-20 \mathrm{~min}$.

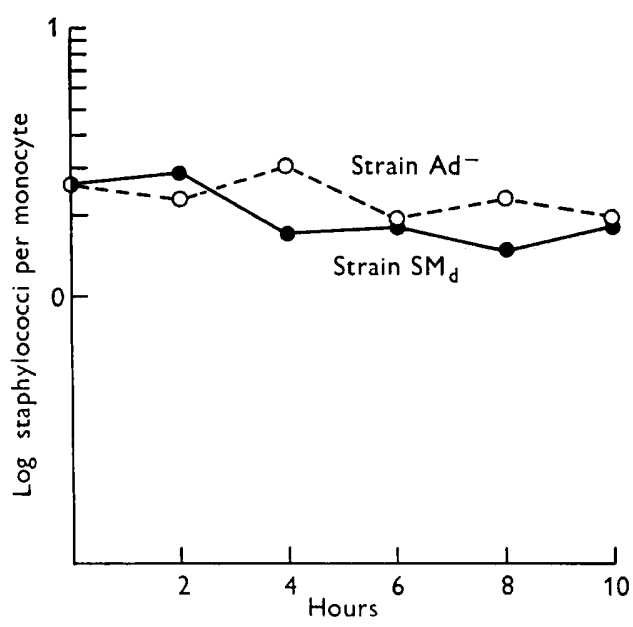

Fig. 5

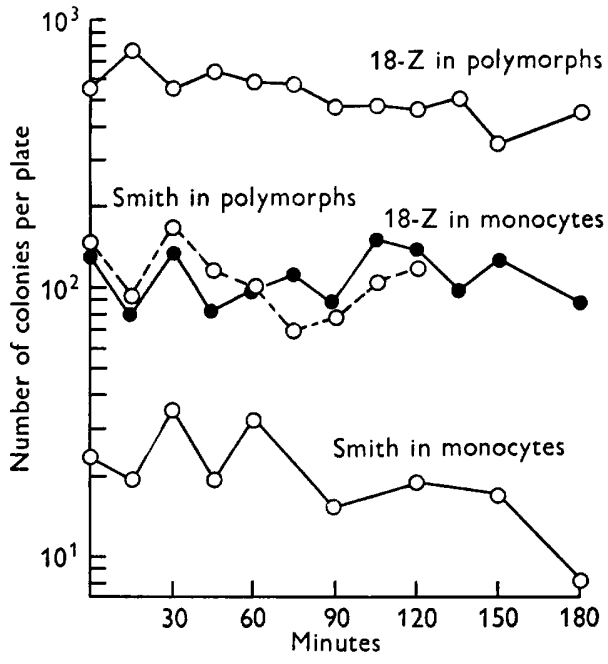

Fig. 6

Fig. 5. Intracellular survival of adenine-requiring ( $\mathrm{Ad}^{-}$) and streptomycin-dependent $\left(\mathrm{SM}_{\mathrm{d}}\right)$ mutants of Staphylococcus aureus $18-\mathrm{Z}$ within rabbit monocytes maintained in plastic chambers.

Fig. 6. Survival of Staphylococcus aureus $18-\mathrm{Z}$ and Smith strains within rabbit polymorphonuclear leucocytes and monocytes by the plate method.

The number of leucocytes in successive samples sometimes decreased for a period, and then again increased after 3-5 hr. This apparently did not reflect a change in total cell population within the flasks since cells were observed to settle along the walls of the vessel. However, such decreases did not occur in all experiments and did not depend on infection since the phenomenon was also observed with non-infected leucocyte cultures (but was not observed with heat killed leucocytes). Apparently the cells adhered to the vessel walls for a time, but later released themselves into the medium. The same phenomenon often occurred in siliconized test tubes (using the Cohn \& Morse procedure); thus the shape of the vessel did not appear responsible for the effect. The mechanism for this reaction is, therefore, not clear.

A series of experiments were performed with rabbit polymorphs or monocytes infected with 18-Z, 18-Z $\mathrm{SM}_{\mathrm{d}}$ and Smith strains. A different rabbit was used as a leucocyte donor in each experiment. 
Figure 7A illustrates the results obtained when rabbit monocytes were infected with a washed $6 \mathrm{hr}$. old culture of $18-\mathrm{Z}$. Fifty $\mu \mathrm{g}$. streptomycin $/ \mathrm{ml}$. were added to the medium $1 \mathrm{hr}$. after the zero time sampling, in order to suppress extracellular multiplication of staphylococci.
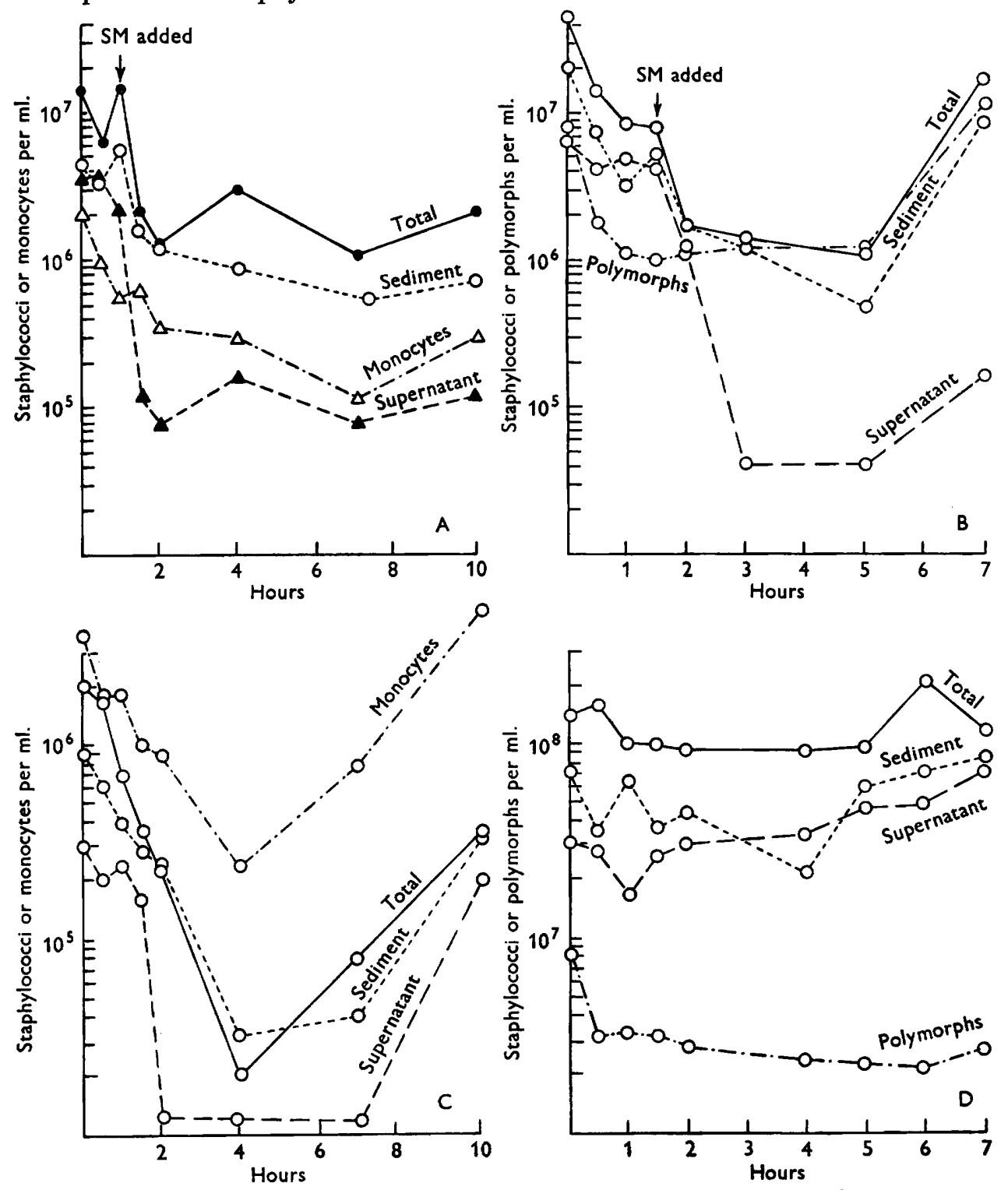

Fig. 7. Survival of staphylococci within leucocytes by the suspended culture procedure. A. Staphylococcus aureus $18-Z$ and rabbit monocytes. B. S. aureus $18-\mathrm{Z}$ and rabbit polymorphs. C. S. aureus $18-\mathrm{Z} \mathrm{SM}_{d}$ and rabbit monocytes. D. S. aureus $18-\mathrm{Z}$ $\mathrm{SM}_{\mathrm{d}}$ and rabbit polymorphs. $\mathrm{SM}=$ streptomycin.

Figure 7B shows the results of an experiment in which a polymorph suspension was infected with a $16 \mathrm{hr}$. culture of $18-\mathrm{Z}, 50 \mu \mathrm{g}$. streptomycin $/ \mathrm{ml}$. was added to the suspended culture medium $1.5 \mathrm{hr}$. later. 
Figure $7 \mathrm{C}$ illustrates the result of an experiment in which rabbit monocytes were infected with a $6 \mathrm{hr}$. culture of the streptomycin-dependent mutant of $18-\mathrm{Z}$ (18-Z, $\mathrm{SM}_{\mathrm{d}}$ ). Figure $7 \mathrm{D}$ shows the findings when rabbit polymorphs were infected with a $16 \mathrm{hr}$. culture of the same organism.
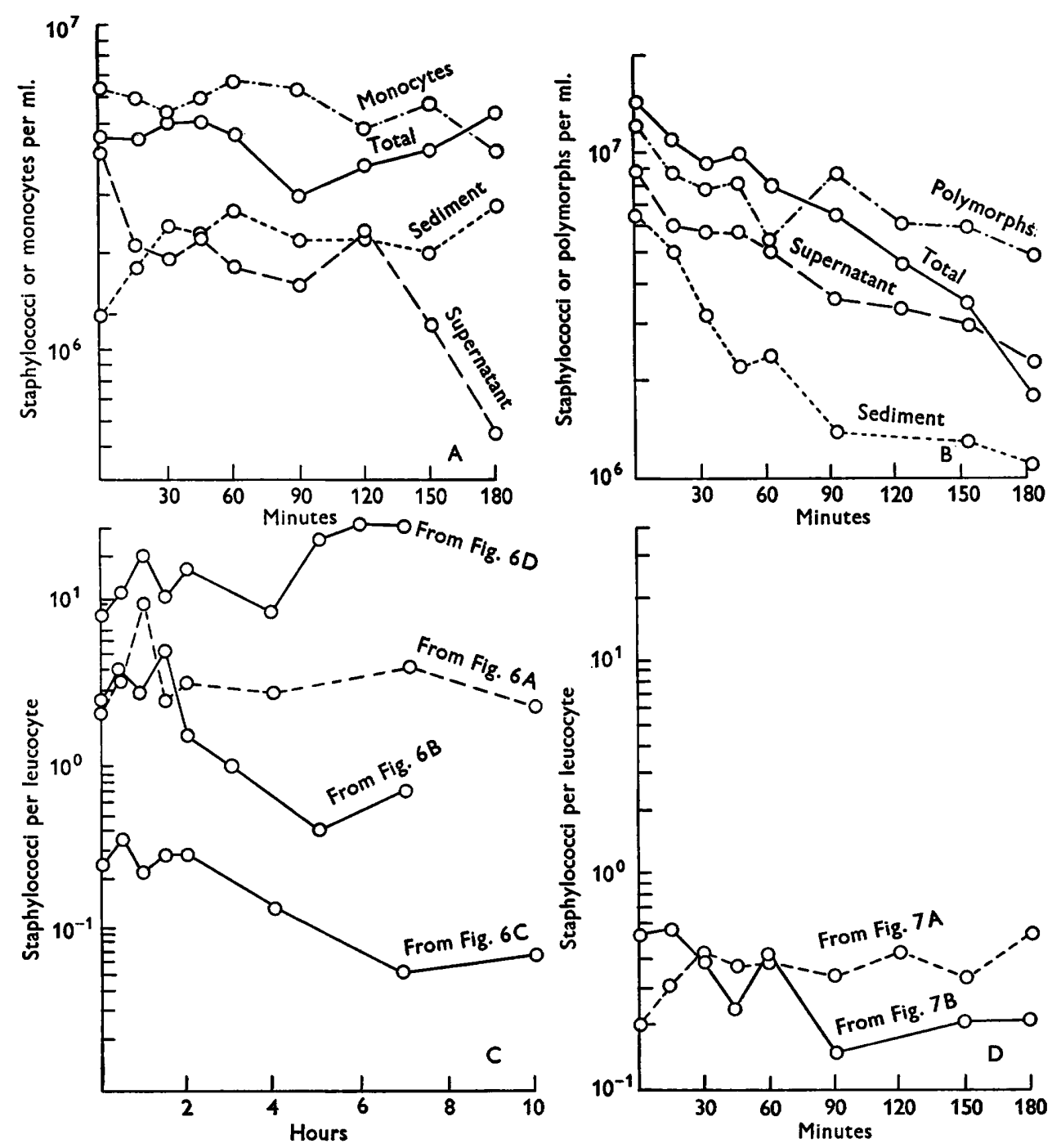

Fig. 8. A. Survival of Staphylococcus aureus Smith within rabbit monocytes. B. Survival of $S$. aureus Smith within rabbit polymorphs. C. Average number of organisms (S. aureus 18-Z and 18-Z, $S_{d}$ ) per cell from data plotted in Fig. 6A-D. D. Average number of organisms (S. aureus Smith) per cell from data plotted in Fig. 7 A-B.

Figure $8 \mathrm{~A}$ and $\mathrm{B}$ illustrate the results of two experiments in which rabbit monocytes or polymorphs were infected with a $20 \mathrm{hr}$. culture of the Smith strain of Staphylococcus aureus, and the infected cell cultures maintained for $3 \mathrm{hr}$. in the absence of antibiotics. 
In the experiments illustrated by Fig. 7B and C, it can be seen that the leucocyte counts decreased temporarily and later increased to the original values during the course of study. However, it may be noted that the number of organisms in the sediment and the total staphylococcal population paralleled the leucocyte population fairly well. Thus the average number of staphylococci per cell (sediment count/leucocyte count) in the cultures usually remained relatively constant for several hr. This is demonstrated in Fig. $8 \mathrm{C}$ and $\mathrm{D}$ where the average number of staphylococci/cell is plotted against time for all the previous mentioned experiments using the suspended culture technique.

\section{DISCUSSION}

Four methods were used to follow the fate of intracellular staphylococci, each method having its advantages and limitations. In the tissue-culture chamber method, the infected leucocytes adhered to cover slips in the same chamber, and the populations of intracellular and extracellular organisms and that of the leucocytes could be followed. However, this method does not permit an independent evaluation of the total population of organisms nor does it allow accurate determination of the intracellular population in the presence of streptomycin during the first hour after phagocytosis.

The Cohn \& Morse method, in addition, permitted the enumeration of the total population of organisms, but continuous phagocytosis in this method might complicate determining the intracellular fate of the organisms.

The suspended culture method is a modification of the Cohn \& Morse method. This procedure had all the advantages of the chamber method and Cohn \& Morse method and in addition made possible sampling of intracellular organisms within $20 \mathrm{~min}$. after phagocytosis in the absence of an excessive extracellular population. Also the packing of leucocytes and organisms permitted rapid phagocytosis without using antibodies. It also allowed all samples to be derived from one vessel. Enumeration of viable leucocytes in each sample gave an opportunity to evaluate the staphylococcal counts in relation to the leucocyte counts.

The plate method has the advantage that it so separates the leucocytes from the extracellular organisms that continual phagocytosis is virtually impossible even without antibiotics.

The results obtained using these methods indicated that Staphylococcus aureus (18-Z and Smith strains) survive in significant numbers within normal rabbit monocytes and polymorphs for several hours. These methods failed to demonstrate any extensive immediate destruction, as reported by Cohn \& Morse (1959).

Rogers \& Melly (1960), using the same procedure and the same organisms, but using normal human blood leucocytes, did not report the same degree of killing as presented in Cohn \& Morse's work. Melly et al. (1960) further studied the intracellular staphylococci by direct observations with phase contrast microscopy and demonstrated some intracellular destruction of staphylococci, but not as much as found by Cohn \& Morse. These latter studies were performed using the leucocytes from a single human donor and thus might not reflect a general phenomenon. This view might be supported by the observed variation in the behaviour of staphylococci within leucocytes derived from different rabbits (Shayegani \& Kapral, 1962). 
It is still difficult at the present time to evaluate the role of leucocytes in the pathogenesis of staphylococcal disease. It appears that the usual Staphylococcus aureus strains (represented by the 18- $Z$ strain) are readily phagocytosed in the presence of normal serum whereas encapsulated strains (such as the Smith strain) may present such an unusual surface to the leucocytes that phagocytosis is reduced in the absence of specific antibodies. What has been termed as normal serum in this study may, however, still contain opsonizing antibodies against $S$. aureus. The possibility exists that there is a widespread occurrence in the serum of many rabbits of antibodies against certain surface antigens commonly associated with $S$. aureus while antibodies against uncommon capsular antigens may be less frequently encountered. The recent report by Cohen, Cowart \& Cherry (1961) demonstrates that antibodies against $S$. aureus are indeed frequently found in the serum of nonimmunized rabbits. Furthermore, it should be mentioned that more recent studies by $\mathrm{Dr}$ Isabel $\mathrm{Li}$ in this laboratory have demonstrated a more pronounced destruction of Staphylococcus aureus phagocytosed in the presence of certainspecificimmune sera than reported in this publication. Studies concerned with the possibility that variations in antisera may account for some of the published quantitative discrepancies are now under way. Nevertheless, after phagocytosis the question still arises whether leucocytes in vivo can effectively destroy Staphylococcus aureus under conditions existing locally at the site of infection. Further work is necessary to answer this question.

This investigation was supported by research grant E-2695 from the National Institutes of Health, United States Public Health Service.

\section{REFERENCES}

Cohen, J. O., Cowart, G. S. \& Cherry, W. B. (1961). Antibodies against Staphylococcus aureus in non-immunized rabbits. J. Bact. 82, 110.

CoHN, Z. A. \& MonSE, S. I. (1959). Interaction between rabbit polymorphonuclear leucocytes and staphylococci. J. exp. Med. 110, 419.

KaPRAL, F. A. \& ShaYegan, M. G. (1959). Intracellular survival of staphylococci. $J$. exp. Med. 110, 123.

MaCKaness, G. B. (1960). The phagocytosis and inactivation of staphylococci by macrophages of normal rabbits. J. exp. Med. 112, 35.

Meliy, M. A., Thomison, J. B. \& Rogers, D. E. (1960). Fate of staphylococci within human leucocytes. J. exp. Med. 112, 1121.

Rogers, D. E. \& Melly, M. A. (1960). Further observation on the behaviour of staphylococci within human leucocytes. J. exp. Med. 111, 533.

Shayegani, M. G. \& Kapral, F. A. (1962). The eventual intracellular destruction of staphylococci by mononuclear cells. J. gen. Microbiol. 29, 637. 